\title{
HACIA UNA ESTRATEGIA ASISTIDA POR ROBOT PARA LA RECUPERACION DE FUNCION MOTORA DE EXTREMIDAD SUPERIOR CON ASPECTOS COGNITIVOS
}

\author{
Edwin Daniel Oña*, Bartek Łukawski, Alberto Jardón, Carlos Balaguer \\ Robotics Lab, Universidad Carlos III de Madrid, Avda. Universidad 30, Leganés (Madrid), España, \\ *eona@ing.uc3m.es; blukawsk@ing.uc3m.es; ajardon@ing.uc3m.es; balaguer@ing.uc3m.es
}

\begin{abstract}
Resumen
A día de hoy, el desarrollo de sistemas robóticos de asistencia en procedimientos de rehabilitación ha sido elevado. Sin embargo, existen ciertas aspectos que limitan un uso más extendido. Una posible causa puede ser que las mejoras en función motora no son traspasadas directamente al desarrollo de actividades de la vida diaria. Esto puede provenir de que las estrategias utilizadas no promueven el entrenamiento de otros factores esenciales en la generación del movimiento, como el cognitivo y perceptual. En este artículo se presenta una estrategia para la rehabilitación de la función motora de la extremidad superior que utiliza un brazo robótico para modelar la interacción con el usuario, añadiendo en la tarea factores para la estimulación cognitiva. El robot es usado para promover la movilización del brazo, mientras se propone al usuario manipular y reconocer objetos. Una webcam colocada en el extremo del robot es utilizada para reconocer los objetos. Además, los movimientos del usuario son monitorizados por una cámara $R G B-D$ para analizar su calidad. En la fase actual de desarrollo se ha completado la integración de los componentes para la estrategia. La estrategia propuesta está orientada a implementar tratamientos personalizables, que incluyan factores cognitivos en el mismo paradigma, y en un entorno más cercano al de la vida diaria.
\end{abstract}

Palabras clave: Rehabilitación, Robot, Extremidad superior

\section{INTRODUCCIÓN}

Las lesiones neurológicas son la principal causa de discapacidad y la segunda causa de muerte a nivel mundial $[17,4]$. Esta situación, sumada al constante crecimiento de la población con edad por encima de 65 años [15] indica que los sistemas de sanidad tienen que enfrentar una alta demanda de atención médica. En este sentido, en las últimas décadas ha habido un alto desarrollo de soluciones basadas en la robótica, para implementar soluciones tecnológicas que contribuyan a optimizar la atención médica $[5,7]$. Uno de los principa- les focos de investigación ha sido la rehabilitación neurológica.

La rehabilitación neurológica se puede definir como un proceso que tiene como objetivo optimizar la participación de una persona en la sociedad y la sensación de bienestar [16]. Esta definición destaca varias características importantes: la rehabilitación no es un tipo particular de intervención; el foco está en el paciente como persona; los objetivos se relacionan con el funcionamiento social, así como con la salud o el bienestar; no es un proceso restringido a pacientes que pueden recuperarse, parcial o completamente, sino que se aplica a todos los pacientes que quedan con problemas a largo plazo.

Las necesidades de los pacientes con desordenes neurológicos son multi-dimensionales (física, $\operatorname{cog}$ nitiva, psicológica, etc.) y pueden ser muy complejas. Además, un mismo tipo de desorden neurológico puede causar problemas a diferentes niveles. Uno de los problemas más comunes son los relacionados con la movilidad de las extremidades. Por dicha razón, uno de los objetivos de la rehabilitación neurológica es recuperar la funcionalidad motora, la cual es esencial para realizar de forma autónoma actividades de la vida diaria.

A pesar del amplio desarrollo en sistemas de este tipo, siguen existiendo vacíos de cara a un uso extendido de soluciones basadas en robots en el ámbito clínico. Una de las posibles causas de esta limitación, es que los actuales sistemas para la recuperación de la función motora se han enfocado principalmente en realizar técnicas de movilización de las extremidades, y basadas en repeticiones. Sin embargo, otros factores como el cognitivo y de percepción, y que participan en la generación del movimiento, han sido abordados en menor medida.

En este artículo, se presenta el diseño y desarrollo de una estrategia de rehabilitación de la función motora de la extremidad superior que incluye: (1) un robot de propósito general para promover la movilización del brazo del usuario, (2) un sistema de seguimiento de los movimientos del usuario basado en una cámara RGB-D, (3) tareas con cier- 
ta carga cognitiva y de percepción mediante manipulación y reconocimiento de objetos, y (4) un método fácil de programación de trayectorias del robot. A continuación, en la sección 1.1 se presentan los fundamentos de la propuesta. La metodología y los elementos utilizados en esta aplicación se describen en la sección 2. El desarrollo e implementación del sistema se presenta en la sección 3. La integración software y hardware del sistema se describe en la sección 4. Las posibilidades de la propuesta se discuten en la sección 5. Finalmente, las conclusiones y trabajos futuros se resumen en la sección 6 .

\subsection{FUNDAMENTOS}

En la actualidad, los sistemas basados en robots desarrollados para la rehabilitación de la función motora del miembro superior se han enfocado principalmente en la interacción física con el usuario y la repetición de movimientos [8]. Independientemente de la morfología del robot utilizado (exoesqueletos o efector-final), los modos de operación pueden ser asistido, activo o pasivo, pudiendo un mismo sistema robótico implementar más de un modo de operación y diferentes combinaciones [1]. Estos modos están basados en replicar las técnicas manuales usadas por los terapeutas para incentivar el re-aprendizaje motor por medio de movilizaciones.

Sin embargo, el aprendizaje motor implica la interacción de múltiples procesos del sistema nervioso central (SNC) y que incluyen factores cognitivos (atención, motivación, planificación, resolución de problemas) y los relacionados con la percepción (información sensorial) [13]. Además, hay que considerar que el movimiento depende de factores externos como el tipo de tarea y el entorno o contexto donde se va a realizar.

De esta manera, el desarrollo de sistemas de asistencia basados en robot deberían considerar factores cognitivos y de percepción para estimular adecuadamente los factores internos del SNC. Además, el entorno o contexto donde se realiza el tratamiento debe acercarse lo más posible a los cotidianos (reales) para maximizar la transferencia de las ganancias motoras a las actividades de la vida diaria [11]. Esta metodología ha sido considerada por el sistema DIEGO ${ }^{\circledR}$ fabricado por Tyromotion [14]. Este sistema de tipo efector-final permite movilizar la extremidad superior en modo pasivo, asistido, activo, y bimanual. Incluye una interfaz de usuario basada en realidad virtual completamente inmersiva, que permite estimular de una mejor manera los aspectos sensoriales y de percepción.

En esta línea, el trabajo presentado en este artícu- lo busca explorar el uso de un robot de propósito general para promover la rehabilitación de la funcionalidad motora, incluyendo factores cognitivos y de percepción en el mismo paradigma. En la sección siguiente, se describe la metodología y elementos utilizados para esta aplicación.

\section{MATERIAL Y MÉTODO}

El propósito final de este trabajo es desarrollar una estrategia de rehabilitación motora mínimamente supervisada, y que incluya factores que promuevan la asimilación de las ganancias obtenidas en la terapia [11]. La estrategia presentada en la Figura 1, considera: (a) la interacción humanorobot (paciente-robot y terapeuta-robot), (b) una mejor elaboración de ejercicios (tarea y entorno) para fomentar las ganancias motoras, y (c) mejorar la capacidad analítica del sistema.

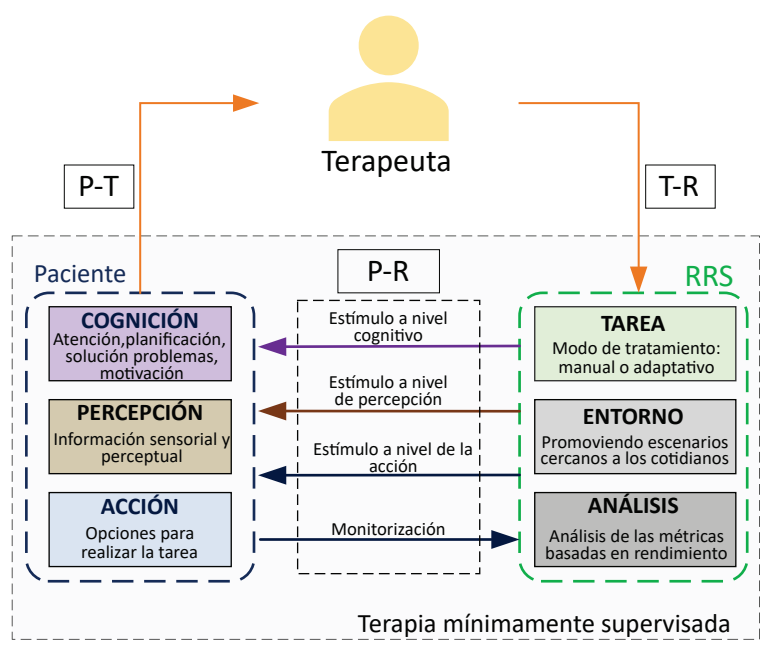

Figura 1: Estrategia para rehabilitación de la extremidad superior asistida por robot.

Se puede observar que los principales actores de nuestra estrategia son el sistema de rehabilitación robótico (RRS por sus siglas en inglés), el paciente y el terapeuta. Este último será quien personalice los ejercicios. De cara a la interacción pacienterobot (P-R), se busca que el RRS promueva la estimulación en el paciente de los factores que participan en la generación del movimiento (cognición, percepción y acción) [13]. Esta estimulación se aborda por medio de tareas que involucren la movilización de la extremidad junto con cierta carga cognitiva, y que se desarrollen en un entorno cercano al de la vida diaria. Finalmente, en línea a incrementar la capacidad analítica del sistema, se hace un seguimiento de los movimientos del paciente. Con respecto a la interacción del robot con el terapeuta (T-R), se ha implementado una método intuitivo de teleoperación para mover el robot y programar los puntos y los objetos a detectar. 
A continuación, se presentan los elementos utilizados para implementar la estrategia propuesta en este artículo.

\subsection{ROBOT GENÉRICO}

Se dispone del robot AMOR fabricado por Exact Dynamics, que es un brazo robótico de propósito general con 7 grados de libertad (GDL). Tiene un diseño esbelto y con poca masa para reducir la inercia de cara a la seguridad. Tiene una carga útil de $2.5 \mathrm{~kg}$ y un alcance de aproximadamente 1 metro.

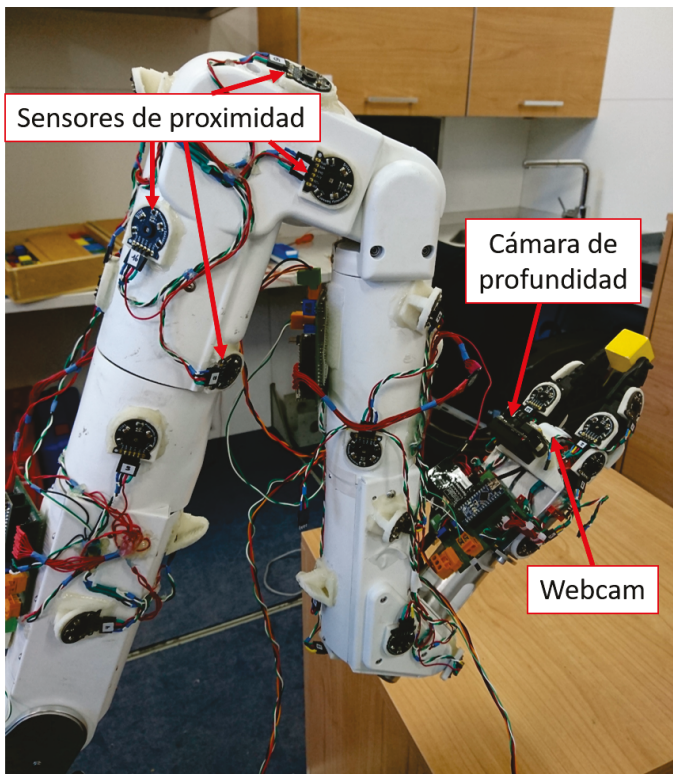

Figura 2: Brazo robótico AMOR

El modelo disponible en el laboratorio cuenta además con 43 sensores de proximidad distribuidos adecuadamente a lo largo del robot para detección de obstáculos.

En la Figura 2, se muestra al robot AMOR con los sensores de proximidad. Además, se pueden apreciar las dos cámaras colocadas en el extremo del brazo, junto a la garra, una cámara RGB y una cámara de profundidad en miniatura. Para su programación, el robot dispone de una propia interfaz de programación de aplicaciones (API por sus siglas en inglés).

\subsection{PERIFÉRICOS DE ENTRADA}

En la Figura 3 se muestran los dispositivos periféricos utilizados para esta aplicación, que son una cámara Kinect ${ }^{\circledR}$ y un joystick SpaceNavigator.

Por un lado, el sensor Kinect ${ }^{\circledR}$ es un controlador originalmente diseñado para su uso en consolas de videojuegos. Este sensor permite a los usuarios in-

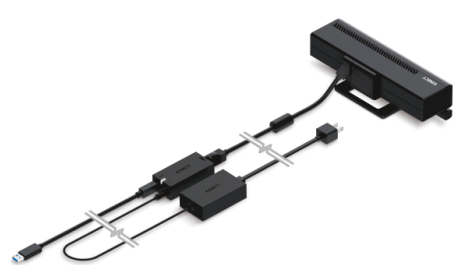

(a) Kinect V2

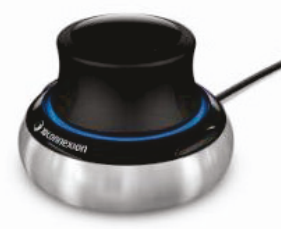

(b) SpaceNavigator
Figura 3: Dispositivos periféricos utilizados.

teractuar sin necesidad de contacto físico directo, por medio de una interfaz natural de usuario. La segunda generación de este sensor ofrece una cámara RGB con una resolución de 1920x1080 píxeles y una cámara de profundidad con un rango de alcance entre 0.5 a 4.5 metros. Una de las principales aplicaciones, fuera del ámbito de los videojuegos, es en la captura de movimientos del cuerpo humano sin necesidad de marcadores. La precisión en el seguimiento de las articulaciones es moderada a excelente, dependiendo de la dimensión del movimiento [12].

Por otro lado, el joystick SpaceNavigator es un dispositivo de 6 GDL, similar a un 'mouse', que permite navegación 3D en aplicaciones de CAD. Además cuenta con dos botones de uso general y programables. La conexión es por medio de un puerto serie USB 2.0.

\section{DISEÑO DEL SISTEMA}

Para la implementación de la estrategia propuesta en este artículo, se ha diseñado una tarea asistida por robot denominada alcanza-y-muestra. Esta tarea se desarrolla en dos fases: preparación y ejecución. En la fase de preparación el terapeuta programa los puntos de interés por donde quiere que pase el robot. Después, se ejecuta la tarea programada, interactuando con el paciente.

Así, el robot AMOR pedirá mediante mensajes de audio que el paciente le muestre un objeto específico de un conjunto de diferentes objetos. El robot se posicionará en diferentes puntos dentro del espacio de alcance del usuario y esperará a que se le muestre el objeto solicitado. Se ha utilizado un conjunto de objetos de diferentes colores y formas (cuadrado, círculo y triángulo). Una cámara RGB, que se encuentra localizada en el extremo del robot, es utilizada para detectar los objetos. Cuando el usuario le muestra al robot el objeto correcto, el robot se desplazará a la siguiente posición y pedirá al usuario que le muestre otro objeto diferente. Durante la interacción paciente-robot, se monitorizan y registran los movimientos de la ex- 
tremidad superior del usuario por medio de un sensor Kinect.

Se puede apreciar que en la tarea propuesta intervienen varios procesos: (1) programación de puntos para trayectoria del robot, (2) reconocimiento de objetos, y (3) monitorización de movimientos del usuario. A continuación se detalla el funcionamiento e implementación de cada proceso.

\subsection{PROGRAMACIÓN DE PUNTOS OBJETIVOS}

Para el desarrollo de la tarea, es necesario definir varios puntos en el espacio donde el robot va a esperar a que el paciente le muestre el objeto solicitado. De cara a definir la trayectoria, se ha implementado una aplicación de usuario en lenguaje $\mathrm{C}++$ para mover el robot, grabar puntos para formar una trayectoria y seleccionar el objeto a identificar en dicho punto. Esta interfaz está orientada al terapeuta.

Por un lado, el fabricante del robot suministra una API y un 'solver'cinemático que permite mover el robot en el espacio articular y cartesiano. Para indicar los desplazamientos se puede utilizar un teclado de ordenador. Sin embargo, este método es poco intuitivo y limita el posicionamiento del robot.

Por tal motivo, para proveer al terapeuta de un modo más intuitivo y rápido para definir varios puntos en el espacio, se ha implementado un controlador para conectar un joystick que permita desplazar y orientar el extremo del robot. En nuestro caso, se ha usado el dispositivo SpaceNavigator de 6 GDL.

La aplicación de usuario conecta de forma transparente el controlador del joystick con el 'solver'cinemático del robot AMOR. Mediante una ventana de diálogo, se pregunta al usuario si desea mover el robot en espacio cartesiano o articular. Una vez colocado en la posición deseada, mediante la ventana de diálogo se confirma que se quiere almacenar las coordenadas de dicho punto.

Por otro lado, durante la grabación de cada punto y antes de pasar al siguiente, usando la misma ventana de diálogo se pide al terapeuta que seleccione el objeto que desea que el robot robot detecte en dicho punto. Repitiendo el proceso anterior se pueden almacenar todos los puntos que sean necesarios para trabajar durante la sesión.

\subsection{RECONOCIMIENTO DE OBJETOS}

Para identificar la forma y el color de los objetos que el usuario le "muestra" al robot, se ha implementado un programa en Matlab para reconocer objetos utilizando la webcam colocada en el extremo del robot. Dicho programa deriva de un trabajo previo [9].

En primer lugar se captura una imagen de la escena que esta viendo la webcam colocada en el extremo del robot. Esta imagen es convertida al espacio de color CIELab. Utilizando como referencia unos marcadores de color previamente calibrados, se clasifican cada uno de los píxeles de la imagen. La clasificación es realizada de acuerdo a la regla del vecino más cercano (kNN Nearest Neighbour en inglés). El algoritmo $\mathrm{kNN}$ asigna a los píxeles de la escena la etiqueta correspondiente al marcador de color más cercano. Esta técnica es simple, eficiente y no requiere de aprendizaje o fase de entrenamiento del algoritmo [2]. En nuestro caso, se calcula la distancia euclídea entre los píxeles de la imagen con respecto de cada marcador de color, utilizando la ecuación 1 con los canales $a^{*}$ y $b^{*}$ del espacio de color CIELab.

$d=\sqrt{\left(a^{*}{ }_{\text {pixel }}-a^{*}{ }_{\text {marker }}\right)^{2}+\left(b^{*}{ }_{\text {pixel }}-b^{*}{ }_{\text {marker }}\right)^{2}}$

Cada píxel de la imagen es etiquetado de acuerdo a la mínima distancia a los marcadores de color. Por ejemplo, si la distancia entre un píxel y el marcador de color rojo es la mínima, dicho píxel es etiquetado como un píxel rojo. Finalmente, la imagen se pasa a blanco y negro y se extraen las características de los objetos en la imagen. En base a su área y forma, se define si el objeto detectado coincide con el objeto que se le pedía al usuario.

Para esa aplicación, se han utilizado objetos con cuatro colores: rojo, verde, amarillo y azul. Por lo tanto, se han definido un total de cuatro marcadores de color en el espacio CIELab. Una de las ventajas de utilizar el espacio de color CIELab es que sus valores de color van de rojo a verde y de amarillo a azul para los canales $a^{*}$ y $b^{*}$, respectivamente. Dada esta oposición en los canales, facilita la identificación de los colores de los objetos en nuestra aplicación [10]. Otra ventaja es que el tercer canal correspondiente a la iluminación está separado, y por lo tanto, hace que la identificación de color sea menos sensible a cambios de iluminación.

\subsection{MONITORIZACIÓN DEL USUARIO}

Para monitorizar los movimientos realizados por el usuario durante el desarrollo de las tareas de alcance, se ha utilizado el sensor Kinect V2 for Windows. Para administrar la adquisición de datos se 


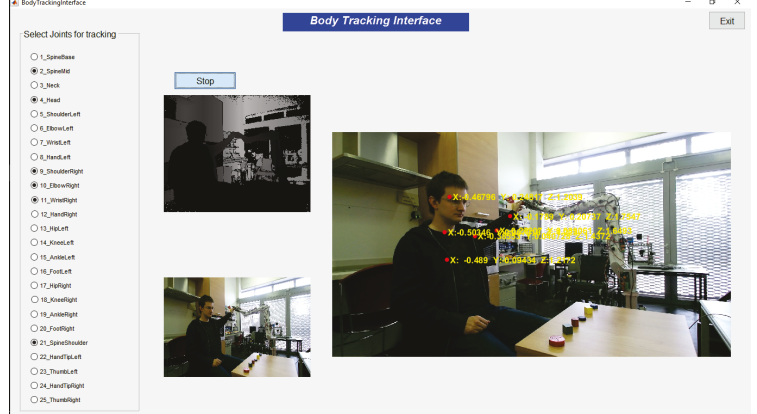

Figura 4: Interfaz para monitorización de los movimientos del usuario.

ha implementado una interfaz gráfica elaborada en MatLab.

En la Figura 4 se puede observar la interfaz de adquisición durante el seguimiento de los movimientos de un usuario. En la parte izquierda de la interfaz, se pueden seleccionar las articulaciones de interés que se quieren monitorizar. Se dispone de un total de 25 articulaciones para elegir, que son las que incorpora el controlador del sensor Kinect V2.

Sin embargo, a pesar de disponer de la opción de monitorizar los movimientos del cuerpo entero del usuario, para ésta aplicación será de interés el rastreo de las articulaciones correspondientes a la extremidad superior.

La interfaz permite visualizar la información de la cámara de color y la de profundidad proporcionadas por el sensor Kinect V2. Además, en la mitad derecha de la interfaz se muestra la imagen de color con información sobrepuesta de las articulaciones rastreadas y su posición. Finalmente, toda la información adquirida es automáticamente almacenada en un fichero .CSV para su posterior análisis.

\section{INTEGRACIÓN SOFTWARE Y HARDWARE}

Como se ha podido apreciar, la estrategia propuesta en este artículo cuenta con diferentes procesos que se deben integrar de forma eficiente para el desarrollo de la tarea. Para no limitar el potencial a la hora de mejorar o modificar el tipo de tratamiento, se ha enfatizado en que la implementación del sistema a nivel software fuese modular y distribuida. En resultado, diferentes procesos independientes pero relacionados, según se detalla en la Figura 5, constituyen una arquitectura distribuida.

A nivel software, la separación de los distintos componentes en atención a sus funcionalidades básicas dota de gran versatilidad al conjunto, haciendo que la funcionalidad sea independiente de un dispositivo concreto ${ }^{1}$. Se pueden distinguir entre tres bloques principales: (a) Adquisición de datos: desde los sensores disponibles tanto para teleoperación (SpaceNavigator, LeapMotion) como para monitorización (Kinect, cámaras RGB, sensores proximidad); (b) Control: procesamiento de las entradas tanto del usuario como del sistema, generación de órdenes de movimiento, cálculo cinemático, y (c) Ejecución del movimiento a nivel articular o cartesiano, ya sea sobre el robot físico o un simulador. Dichos componentes, también llamados módulos, pueden ejecutarse en diferentes máquinas comunicadas entre sí dentro de una misma red local.

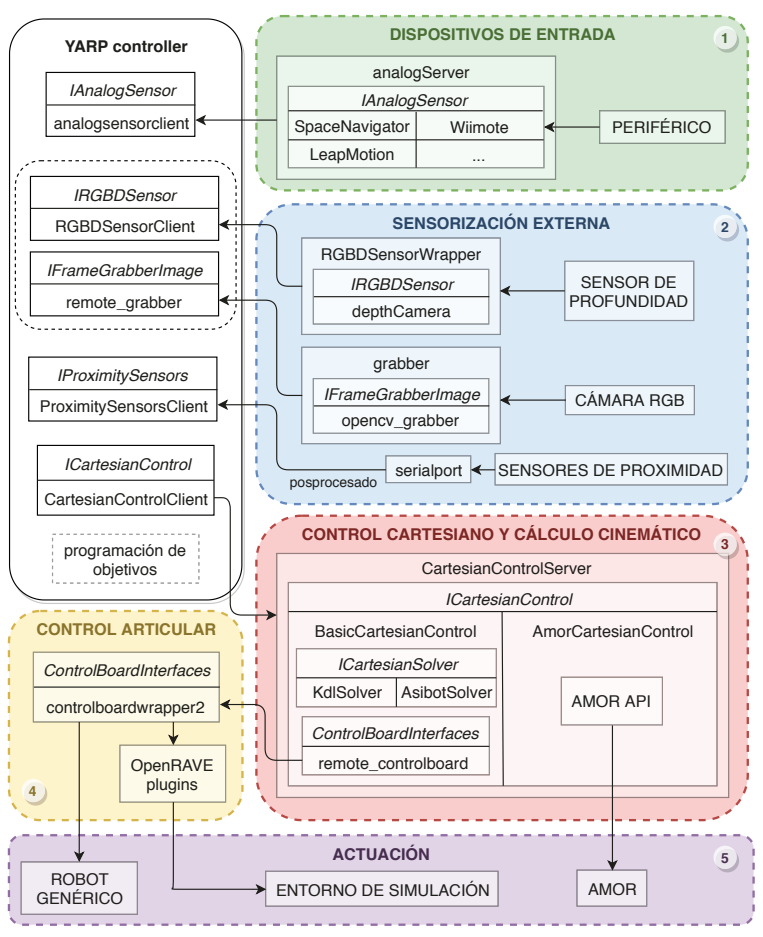

Figura 5: Arquitectura software para la integración de los módulos.

En esta arquitectura, se ha empleado el "framework" de código abierto YARP a fin de conseguir la modularidad y distribución deseadas. Este "framework", centrado en el desarrollo de aplicaciones robóticas, brinda una serie de herramientas multiplataforma que agilizan la implementación de soluciones centradas en la actuación de los motores, el registro de datos mediante sensorización externa o interna, el mapeado y navegación del robot o la captura de entradas analógicas de tipo joystick, por citar algunos ejemplos. La comunicación entre los diferentes módulos es realizada mediante el protocolo TCP/IP. El lenguaje de programación acogido es $\mathrm{C}++$, si bien YARP expone sus inter-

\footnotetext{
${ }^{1}$ Repositorio disponible en este link
} 
faces en lenguajes de más alto nivel como Python.

A nivel hardware, los componentes físicos empleados en el sistema y su disposición se muestran en la Figura 6. Para la implementación de la tarea alcanza-y-muestra se han utilizado el robot AMOR con una cámara RGB colocada en el extremo de su actuador, un sensor Kinect, un monitor LCD para mostrar lo que detecta la cámara del extremo del robot, un conjunto de objetos con diferentes formas y colores.

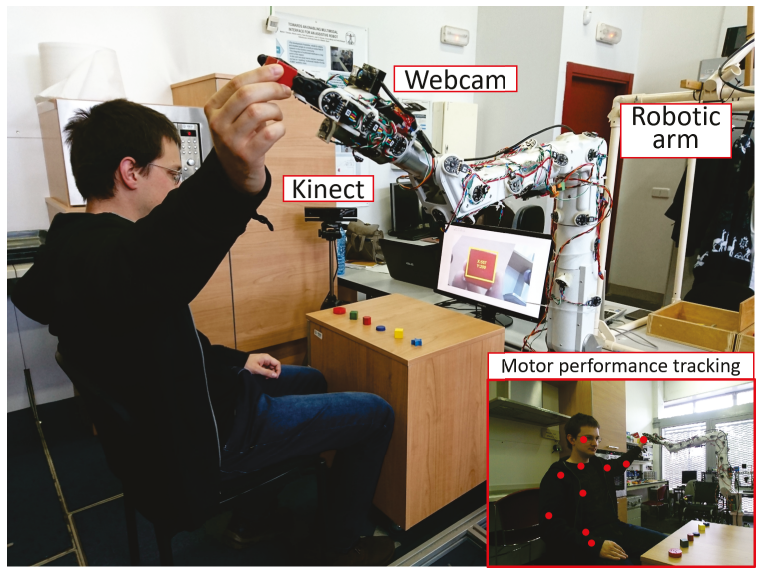

Figura 6: Disposición física de los componentes usados en la tarea alcanza-y-muestra.

El entorno de trabajo para la preparación de la tarea es una réplica a tamaño real de una cocina que cuenta con fregadero, horno, cocina, lavavajillas, nevera, microondas, y estanterías superiores.

En la Figura 6 también se puede apreciar la disposición de los componentes físicos para validar la integración de los diferentes procesos. En la esquina inferior derecha se muestra una captura sincronizada de la monitorización de movimientos con el sensor Kinect.

\section{DISCUSIÓN}

La complejidad de los procesos involucrados durante el ciclo de rehabilitación neurológica hacen que el desarrollar sistemas adecuados y efectivos en este campo sea altamente desafiante. Por un lado, la variabilidad en el grado y tipo de problemas que pueden provocar las lesiones neurológicas, hacen que las necesidades de tratamiento sean diferentes para cada paciente. En este sentido, los actuales dispositivos de rehabilitación basados en robot implementan estrategias para adaptar los parámetros de los sistemas y variar el tratamiento (intensidad, duración, modo de operación, etc). Esta personalización en el tratamiento parece ser adecuada de cara a cubrir las variables necesidades en los procedimientos de rehabilitación neurológica. Por otro lado, existe evidencia de que los tratamientos basados en robot mejoran la funcionalidad motora. Sin embargo, existe también evidencia de que las mejoras en la función motora obtenidas con el tratamiento basado en robot a menudo no repercuten de forma significativa en el desarrollo de las actividades de la vida diaria $[3,6]$. Por ello es necesario el desarrollo de sistemas que mejoren dicha condición.

Una posible solución a esa limitación en la transferencia de ganancias motoras a actividades de la vida diaria puede ser potenciar los factores que participan en la generación del movimiento (relacionados con el propio paciente, con el entorno y la tarea). Así, se ha diseñado una estrategia para intentar reducir las limitaciones anteriormente descritas.

Además, en dirección a mejorar la capacidad analítica del sistema, se ha incluido un sistema para la monitorización no invasiva de los movimientos del paciente. En base a los datos registrados por el sensor Kinect y los tiempos que el usuario tarda en mostrar correctamente el objeto al robot, se puede analizar el grado de dificultad y el rango de movimiento del usuario. El disponer de información objetiva basada en el rendimiento del paciente puede permitir que se incremente la autonomía del sistema.

En la fase actual de desarrollo se han completado la integración y funcionamiento modular de los componentes necesarios para nuestra estrategia de rehabilitación. Por un lado, se han implementado tres procesos que participan activamente en la estrategia propuesta: la programación de puntos objetivos, el reconocimiento de objetos, y la monitorización del usuario.

La integración de cada proceso dentro del mismo paradigma experimental ha sido completada satisfactoriamente en el laboratorio. Se ha podido comprobar que la comunicación entre módulos por medio de YARP es efectiva y permite abstraer la programación tanto del sistema operativo como de un hardware específico. De esta manera, se puede utilizar diferentes dispositivos para una misma funcionalidad (Ejm.: SpaceNavigator, LeapMotion, o WiiMote para guiar al robot).

\section{CONCLUSIONES Y TRABAJOS FUTUROS}

En este artículo se ha presentado el diseño de una estrategia para rehabilitación de la función motora de la extremidad superior asistida por robot. Se ha propuesto un paradigma en el que se trabaje los factores que están involucrados en la generación del movimiento, a saber, procesos internos del propio paciente (cognitivos, perceptuales y de 
acción), el tipo de tarea, y el entorno o contexto donde se realiza.

En nuestra propuesta, el diseño de la tarea incluye factores que pueden promueven la estimulación de los procesos cognitivos y de percepción ya que se utilizan diferentes objetos que el usuario debe reconocer, manipular y desplazar a diferentes posiciones espaciales. En esta fase de desarrollo, se ha comprobado la integración de los diferentes elementos que componen la tarea, validando la modularidad de nuestra propuesta.

Los trabajos futuros consideran trabajar con objetos cotidianos del entorno tales como cubiertos, tazas, etc. Otra línea de interés es el uso de un brazo robótico que permita un mayor grado de interacción física para incluir procedimientos de movilización de las extremidades. Actualmente se está trabajando con el robot colaborativo LBR IIWA de KUKA. Gracias a la modularidad de nuestra estrategia, se espera que la integración de dicho robot en nuestro paradigma sea sencilla.

Esta línea de trabajo busca desarrollar sistemas de rehabilitación de la función motora, que sean más autónomos y objetivos, y que permitan que las ganancias motoras sean sostenidas y transferibles a actividades de la vida diaria.

\section{Agradecimientos}

The research leading to these results has been funded in part by the Spanish Ministry of Economy and Competitiveness via the ROBOESPAS project (DPI2017-87562-C2-1-R), and in part by the RoboCity2030-DIH-CM, Madrid Robotics Digital Innovation Hub, S2018/NMT-4331, which is funded by "Programas de Actividades I+D en la Comunidad de Madrid" and co-funded by Structural Funds of the EU.

\section{English summary}

\section{TOWARDS A ROBOT-AIDED STRATEGY FOR RECOVERY OF UPPER LIMB MOTOR FUN- CTION ADDING COGNITIVE FACTORS}

\author{
Abstract \\ At present, the development of robot-aided \\ systems in rehabilitative procedures has \\ been high. However, there are aspects that \\ limit extended use. A possible cause is that \\ motor gains usually are not transferred to \\ the performance of daily living activities. \\ This fact likely is due to current strate-
}

gies are enough not promoting the training of other relevant aspects involved in motion generation, such as cognition and perception. This paper presents a strategy for the rehabilitation of upper limb motor function using a robotic arm for modelling the human-robot interaction and adding a cognitive load. The robot is used for promoting the user's arm mobilisation and to propose identification and manipulation of objects. A webcam fixed on the robot gripper is employed to identify the objects. User's movements are registered via an $R G B-D$ camera. In the current development stage, the integration of the strategy components has been successfully performed. The proposed strategy aims to implement customisable treatments that include cognitive aspects in the same paradigm, and into environments similar to the quotidian ones.

Keywords: Rehabilitation, Robot, Upper extremity.

\section{Referencias}

[1] A. Basteris, S. M. Nijenhuis, A. H. Stienen, J. H. Buurke, G. B. Prange, and F. Amirabdollahian. Training modalities in robotmediated upper limb rehabilitation in stroke: a framework for classification based on a systematic review. Journal of neuroengineering and rehabilitation, 11(1):111, 2014.

[2] O. Boiman, E. Shechtman, and M. Irani. In defense of nearest-neighbor based image classification. In 2008 IEEE Conference on Computer Vision and Pattern Recognition, pages 1-8. IEEE, 2008.

[3] S. E. Fasoli, H. I. Krebs, J. Stein, W. R. Frontera, R. Hughes, and N. Hogan. Robotic therapy for chronic motor impairments after stroke: Follow-up results. Archives of physical medicine and rehabilitation, 85(7):1106-1111, 2004.

[4] V. L. Feigin, A. A. Abajobir, K. H. Abate, F. Abd-Allah, A. M. Abdulle, S. F. Abera, G. Y. Abyu, M. B. Ahmed, A. N. Aichour, I. Aichour, et al. Global, regional, and national burden of neurological disorders during 1990-2015: a systematic analysis for the global burden of disease study 2015. The Lancet Neurology, 16(11):877-897, 2017. 
[5] A. Jardón, A. Giménez, R. Correal, S. Martínez, and C. Balaguer. Asibot: robot portátil de asistencia a discapacitados. concepto, arquitectura de control y evaluación clínica. Revista Iberoamericana de Automática e Informática industrial, 5(2): 48-59, 2008.

[6] A. C. Lo, P. D. Guarino, L. G. Richards, J. K. Haselkorn, G. F. Wittenberg, D. G. Federman, R. J. Ringer, T. H. Wagner, H. I. Krebs, B. T. Volpe, et al. Robot-assisted therapy for long-term upper-limb impairment after stroke. New England Journal of Medicine, 362 (19):1772-1783, 2010.

[7] A. Mancisidor, A. Zubizarreta, I. Cabanes, P. Bengoa, and J. H. Jung. Dispositivo robótico multifuncional para la rehabilitación de las extremidades superiores. Revista Iberoamericana de Automática e Informática industrial, 15(2):180-191, 2018.

[8] E. D. Oña, R. Cano de la Cuerda, P. SánchezHerrera, C. Balaguer, and A. Jardón. A review of robotics in neurorehabilitation: Towards an automated process for upper limb. Journal of Healthcare Engineering, 2018 (Article ID 9758939):1-19, 2018.

[9] E. D. Oña, A. Jardón, and C. Balaguer. The automated box and blocks test an autonomous assessment method of gross manual dexterity in stroke rehabilitation. In Y. Gao, S. Fallah, Y. Jin, and C. Lekakou, editors, Towards Autonomous Robotic Systems, pages 101-114, Cham, 2017. Springer.

[10] E. D. Oña, P. Sánchez-Herrera, A. CuestaGómez, S. Martinez, A. Jardón, and C. Balaguer. Automatic outcome in manual dexterity assessment using colour segmentation and nearest neighbour classifier. Sensors, 18 (9):2876, 2018.

[11] E. D. Oña, J. M. Garcia-Haro, A. Jardón, and C. Balaguer. Robotics in health care: Perspectives of robot-aided interventions in clinical practice for rehabilitation of upper limbs. Applied Sciences, 9(13):2586, 2019.

[12] K. Otte, B. Kayser, S. Mansow-Model, J. Verrel, F. Paul, A. U. Brandt, and T. SchmitzHübsch. Accuracy and reliability of the kinect version 2 for clinical measurement of motor function. PloS one, 11(11):e0166532, 2016.

[13] A. Shumway-Cook and M. H. Woollacott. Motor control: translating research into clinical practice. Lippincott Williams \& Wilkins, Philadelphia, PA, 4 edition, 2007.

[14] Tyromotion Inc. Diego system, 2007. URL https://tyromotion.com/en/produkte/ diego/. (Último acceso: 30 Junio 2019).
[15] United Nations, Department of Economic and Social Affairs, Population Division (2017). World Population Ageing 2017. United Nations, 2017. https: //www.un.org/en/development/desa/ population/theme/ageing/WPA2017 asp (Accessed October 2018).

[16] D. Wade. Principles of neurological rehabilitation. In Measurement in Neurological Rehabilitation. Oxford University Press, 1992. ISBN 9780192619549.

[17] World Health Organization. Neurological disorders: public health challenges. WHO Press, 2006. http://www. who.int/mental_ health/neurology/neurodiso/en/ (Accessed October 2018).

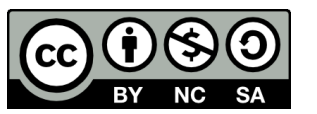

(c) 2019 by the authors. Submitted for possible open access publication under the terms and conditions of the Creative Commons Attribution CC BY-NC-SA 4.0 license (https://creativecommons.org/licenses/by-ncsa/4.0/deed.es). 